\title{
Effects of Weekly Teriparatide Administration for Vertebral Stability and Bony Union in Patients with Acute Osteoporotic Vertebral Fractures
}

\author{
Kazuma Kitaguchi $^{1,2}$, Masafumi Kashii ${ }^{1,2}$, Kosuke Ebina ${ }^{2}$, Satoru Sasaki ${ }^{3}$, \\ Yasunori Tsukamoto ${ }^{4}$, Hideki Yoshikawa ${ }^{2}$, Tsuyoshi Murase ${ }^{2}$ \\ ${ }^{1}$ Department of Orthopedic Surgery, Toyonaka Municipal Hospital, Toyonaka, Japan \\ ${ }^{2}$ Department of Orthopedic Surgery, Faculty of Medicine, Osaka University Graduate School of Medicine, Suita, Japan \\ ${ }^{3}$ Department of Orthopedic Surgery, Kyoritsu Hospital, Kawanishi, Japan \\ ${ }^{4}$ Department of Orthopedic Surgery, North Osaka Police Hospital, Ibaraki, Japan
}

Study Design: An open-label, non-randomized prospective study.

Purpose: Teriparatide (TPTD) is known to be an antiosteoporotic agent that may accelerate the healing of fractures. This study was designed to investigate the effect of once-weekly TPTD administration on vertebral stability and bony union after acute osteoporotic vertebral fracture (OVF).

Overview of Literature: Once-weekly TPTD administration can lead to early vertebral stability and promote bony union of fractured vertebrae in patients with severe osteoporosis.

Methods: Forty-eight subjects with acute OVF were assigned to receive activated vitamin D3 and calcium supplementation or onceweekly subcutaneous injection of TPTD $(56.5 \mu \mathrm{g})$ in combination with activated vitamin D3 and calcium supplementation for 12 weeks. Vertebral stability was assessed using lateral plain radiography. Vertebral height at the anterior location (VHa) and the difference in $\mathrm{VHa}\{\triangle \mathrm{VHa}=\mathrm{VHa}$ (supine position)-VHa (weight-bearing position)\} were measured at baseline and 12 weeks after starting treatment. Bony union was defined as the absence of a vertebral cleft or abnormal motion ( $\triangle \mathrm{VHa}>2 \mathrm{~mm}$ ).

Results: Although not significant, $\triangle \mathrm{VHa}$, indicating vertebral stability, tended to be lower in the TPTD group at 12 weeks ( $p=0.17)$. As for subjects with severe osteoporosis, $\triangle \mathrm{VHa}$ at 12 weeks was significantly lower in the TPTD group than in the control group (mean $\triangle V$ Ha: control group, $3.1 \mathrm{~mm}(\mathrm{n}=15)$; TPTD group, $1.4 \mathrm{~mm}(\mathrm{n}=16) ; p=0.02)$. The rate of bony union was significantly higher in the TPTD group than in the control group (control group, $40 \%$; TPTD group, $81 \% ; p=0.03$ ).

Conclusions: Once-weekly TPTD administration may facilitate early bony union after acute OVF accompanied by severe osteoporosis.

Keywords: Vertebral fracture; Osteoporosis; Bony union; Teriparatide; Vertebral height

\section{Introduction}

Osteoporotic vertebral fracture (OVF) is the most fre- quent fragility fracture among patients with osteoporosis. OVF causes acute back pain, and secondary vertebral deformity after OVF results in chronic back pain. Poor

Received Dec 3, 2018; Revised Jan 16, 2019; Accepted Feb 7, 2019

Corresponding author: Masafumi Kashii

Department of Orthopedic Surgery, Toyonaka Municipal Hospital, 4-14-1 Shibaharacho, Toyonaka, Osaka 560-8565, Japan

Tel: +81-06-6843-0101, Fax: +81-06-6858-3531, E-mail: mkashii-osk@umin.ac.jp 
postural deformity, dysfunction in digestive organs, and pulmonary hypofunction caused by progressive vertebral kyphotic deformity result in decreased participation in the activities of daily living and reduced quality of life among elderly patients [1-3]. The majority of OVF cases are successfully treated with a conservative regimen of bed rest and spinal orthosis. However, delayed vertebral union or nonunion may occur. Delayed union or nonunion leads to long-term physical deterioration [4,5]. The most extreme cases may result in osteoporotic vertebral collapse with neurological deficit [6].

Teriparatide (TPTD), which became available for use in Japan in 2010, promotes cortical and cancellous bone formation and has been widely used for the treatment of severe osteoporosis with high risk of fracture. This agent also garnered a great deal of attention in the field of fracture treatment $[7,8]$. The administration of TPTD to patients with acute OVF may prevent subsequent osteoporotic fractures and promote the healing of fractures. However, few reports have investigated the effects of TPTD for fracture repair in humans [9-11]. No previous report has investigated the effects of TPTD on the healing of fractures after acute OVF because no clear criteria or definitions have been established for bony union after acute OVF using plain radiographs $[7,8]$.

The purpose of the present study was to investigate the effect of once-weekly TPTD administration on vertebral stability and bony union after acute OVF using quantitative criteria.

\section{Materials and Methods}

\section{Study design and subjects}

This was an open-label, non-randomized prospective study. With our protocol, subjects with acute OVF were assigned to the control group and received activated vitamin D3 and calcium supplementation. Control subjects did not receive any antiosteoporotic drugs during the investigation period. Therefore, considering ethical aspects, we recommended once-weekly TPTD administration to all subjects at the time of their initial examination and assigned those who refused TPTD administration to the control group.

We enrolled subjects with acute OVF registered between January 1, 2014 and December 31, 2016 at two facilities associated with the Department of Orthopedics at
Osaka University Graduate School of Medicine. OVF was diagnosed in cases with no history of trauma or a result of low-energy trauma such as a fall on the ground. Acute OVF was defined as occurring within 1 week after injury or the onset of symptoms. The study was conducted in accordance with the Declaration of Helsinki and with the approval of the ethics review boards of all participating facilities (approval no., 12410-2; Osaka University Graduate School of Medicine). All subjects in the study provided written informed consent.

During the investigation period, subjects in the control group received daily oral alfacalcidol $0.5 \mu \mathrm{g}$ and oral calcium lactate $2 \mathrm{~g}$ (or oral calcium aspartate $600 \mathrm{mg}$ ). Those in the TPTD group also received once-weekly TPTD acetate (Teribone; Asahi Kasei Pharma Co. Ltd., Tokyo, Japan) $56.5 \mu$ g, which was administered subcutaneously. All subjects were hospitalized and instructed to remain at rest in a lateral decubitus position, rather than a supine position. After their pain was alleviated and they were able to move in bed, they were fitted for a spinal brace and forced to continuously wear a spinal brace for approximately 3 months during the study period.

\section{Inclusion and exclusion criteria}

Subjects with acute OVF were eligible for the study, regardless of having undergone prior treatment for osteoporosis. All subjects who were hospitalized with acute back pain underwent whole-spine magnetic resonance imaging (MRI). The presence of low T1 signal intensities on T1weighted images and high signal intensities on short-tau inversion recovery sequences were diagnosed as acute OVF. To ensure that all subjects exclusively had primary osteoporosis, subjects with secondary osteoporosis, including rheumatoid arthritis or glucocorticoid-induced osteoporosis, were excluded from the study. No subjects enrolled in this study had pathological fractures with primary spinal tumors, metastatic spinal tumors, myeloma, spinal infections, or metabolic bone diseases such as osteomalacia and hyperparathyroidism. Subjects with acute burst fracture with injury to the posterior wall were also excluded.

\section{Clinical assessment}

Within 3 days of admission to the hospital, all subjects underwent bone mineral density (BMD) measurements, 
whole-spine MRI, and biochemical examination of blood and bone turnover markers. BMD measurements were performed using dual-energy X-ray absorptiometry (GE Lunar Prodigy, Bedford, MA, USA) on lumbar spine (L2-4) and femoral neck. A BMD T-score was calculated for all measured sites. Blood samples were collected, and routine serum chemistry determinations were performed with standard automated techniques. We measured levels of serum calcium, alkaline phosphatase, creatinine, estimated glomerular filtration rate, and intact parathyroid hormone. Serum intact $\mathrm{N}$-terminal propeptide of type I procollagen and tartrate-resistant acid phosphatase $5 \mathrm{~b}$ were also assessed, as markers of bone turnover.

\section{Assessment of vertebral stability and bony union}

Previous studies comparing plain radiographs of subjects with acute OVF in weight-bearing (standing or seating) and non-weight-bearing positions (lateral decubitus or supine position) have reported that a comparison of ver- tebral morphology and vertebral height at the anterior location $(\mathrm{VHa})$ in each position is effective in diagnosing acute OVF [12-15] and assessing bony union [16].

Lateral plane radiographs were taken, with a focus on the fractured vertebral body in weight-bearing (standing or seating if subjects were unable to stand) and nonweight-bearing (supine) positions. On the lateral radiograph, two points were hand selected and marked on each vertebra. These two points were placed at the most anterior-superior and anterior-inferior endplate margins, respectively. VHa was set as the distance in millimeters between identical points on the superior and inferior endplates at the anterior location (Fig. 1). VHa in both positions was measured at baseline and 12 weeks after starting the treatment. The difference in $\mathrm{VHa}\{\Delta \mathrm{VHa}=\mathrm{VHa}$ (supine position)-VHa (weight-bearing position)\} was calculated. The difference in VHa was taken as a measure of vertebral stability and compared between groups. The rate of vertebral collapse $\{\mathrm{VHa}$ (weight-bearing position, baseline)-VHa (weight-bearing position, 12 weeks after
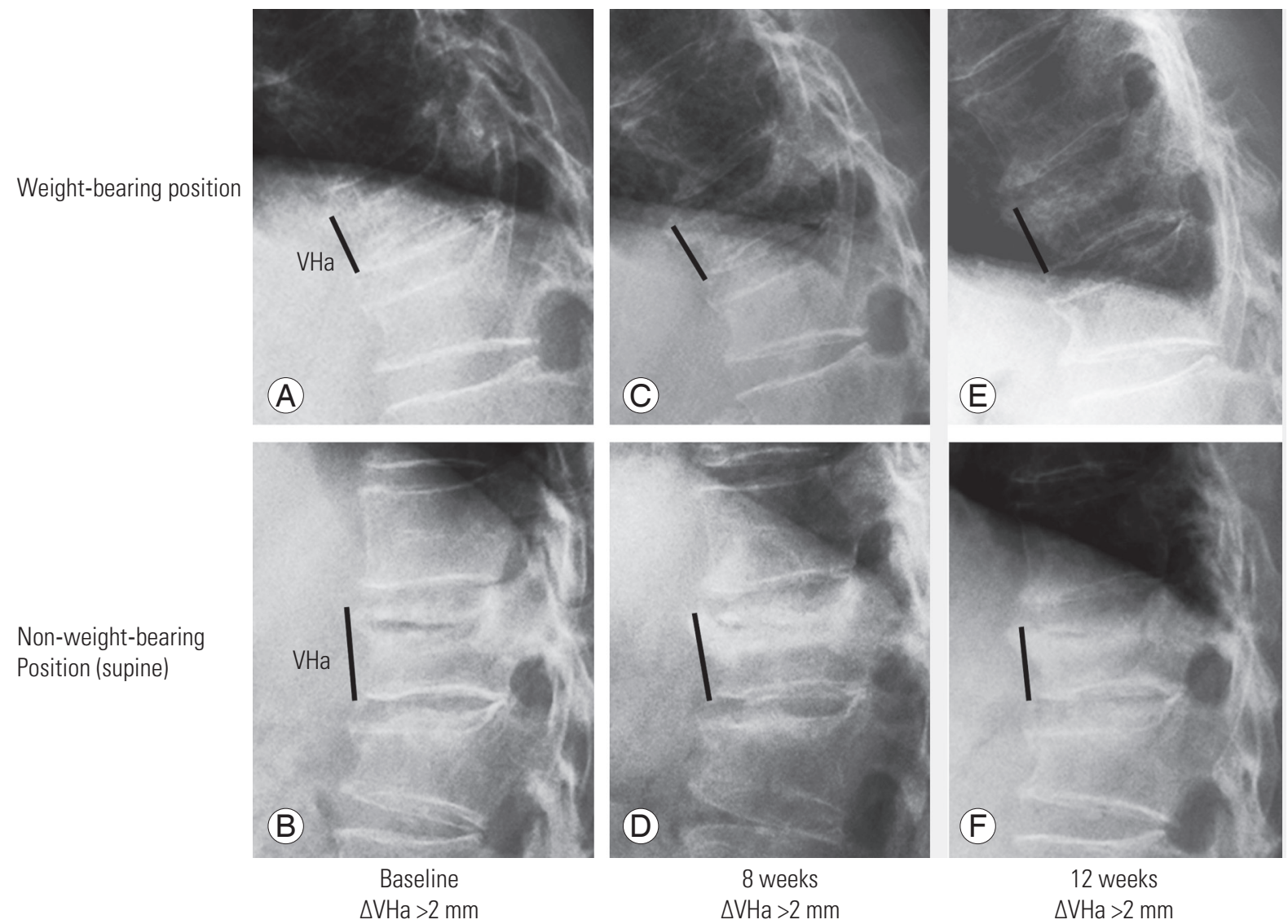

Fig. 1. Chronological assessment of vertebral stability and bony union after acute osteoporotic vertebral fracture. VHa was measured in both weight-bearing and non-weight-bearing positions at baseline $(\mathbf{A}, \mathbf{B})$ as well as after $8(\mathbf{C}, \mathbf{D})$ and 12 weeks $(\mathbf{E}, \mathbf{F})$. The difference in VHa was calculated. VHa, vertebral height at the anterior location. 
starting treatment) divided by $\mathrm{VHa}$ (weight-bearing position, baseline)\} was also calculated.

Based on the results of previous studies, bony union was defined as the absence of a vertebral cleft or abnormal motion $(\Delta \mathrm{VHa}>2 \mathrm{~mm})[12,13,16]$. Rates of bony union were compared between groups at 12 weeks. X-ray measurements and grading via the semi-quantitative (SQ) method were performed by a single spine surgeon [17], who was blinded to each subject's group. The number of prevalent OVFs from T4 to L4 was investigated with the use of spinal MRI images. Acute burst fracture with a protruding bony fragment was excluded.

\section{Reproducibility of the vertebral height at the anterior location}

To assess the reproducibility of measurements of $\mathrm{VHa}$ and $\triangle \mathrm{VHa}$, intraobserver and interobserver intraclass correlation coefficients (ICC) were calculated. For analysis of intraobserver reliability, a spinal surgeon measured both $\mathrm{VHa}$ and $\Delta \mathrm{VHa}$ in 15 patients with vertebral fractures (30 fractured vertebral bodies), in the supine position and the weight-bearing position. Measurements were obtained twice, with a 2-week interval. For the analysis of interobserver reliability, two spinal surgeons and a general orthopedic surgeon measured these vertebral fractures once in the same way. Intraobserver and interobserver ICC for VHa were 0.988 (95\% confidence interval [CI], $0.976-0.994)$ and 0.983 (95\% CI, 0.96-0.992), respectively. Intraobserver and interobserver ICC for $\triangle \mathrm{VHa}$ were 0.925 (95\% CI, 0.798-0.925) and 0.903 (95\% CI, 0.788-0.963), respectively. The standard error for $\mathrm{VHa}$ and $\triangle \mathrm{VHa}$ (intraobserver) was $0.48 \mathrm{~mm}$ and $0.66 \mathrm{~mm}$, respectively.

\section{Statistical analysis}

Statistical analysis was performed using IBM SPSS software ver. 22.0 (IBM Corp., Armonk, NY, USA). All data reported here are expressed as means and standard error (SE). Statistical analysis of comparisons between groups was performed using Mann-Whitney's $U$-test, chi-square test, and Fisher's exact test. Changes from baseline were statistically analyzed using Wilcoxon's signed-rank test. Probability values lower than 0.05 were considered significant.

\section{Results}

We obtained consent from 71 subjects with acute OVF (36 and 35 in the control and TPTD groups, respectively). Subjects who transferred to a different hospital during the study period and those who discontinued treatment with once-weekly TPTD due to adverse effects were excluded

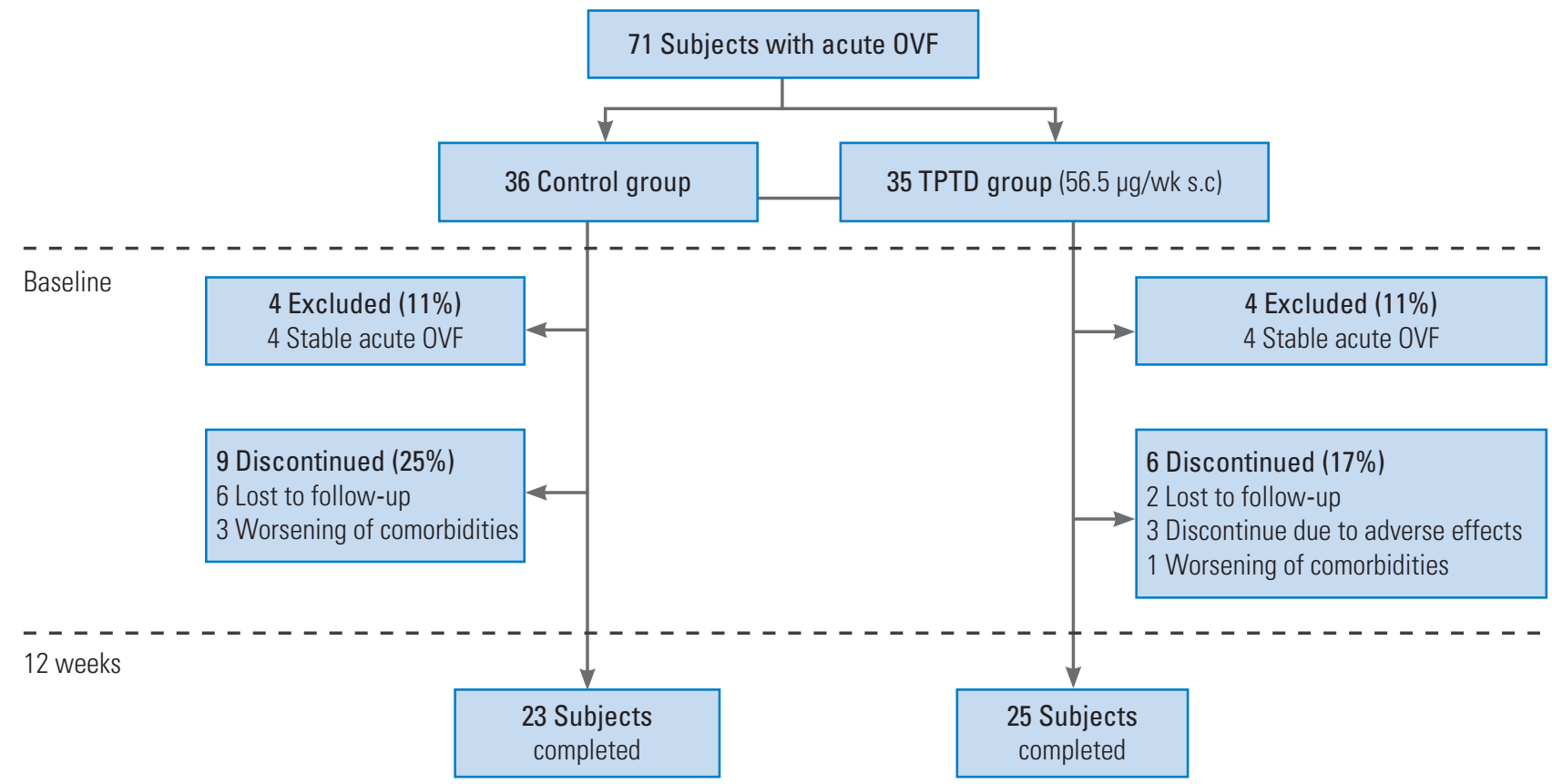

Fig. 2. Study flow-chart and disposition of subjects in the control group and the weekly TPTD use group. OVF, osteoporotic vertebral fracture; TPTD teriparatide. 
from the study. We also excluded eight subjects with stable OVF with a $\triangle \mathrm{VHa}$ of $2 \mathrm{~mm}$ or less at baseline (four subjects in each group). The remaining cohort comprised 23 subjects in the control group and 25 in the TPTD group (Fig. 2). The baseline characteristics in both groups are shown in Table 1. Although the trend was not significant, the TPTD group tended to have a higher rate of prevalent OVFs with severe vertebral deformity and a higher percentage of women. Rates of osteoporosis treatment at baseline tended to be higher in the TPTD group (control, $17 \%$; TPTD, $40 \%$; $p=0.09$ ), and oral bisphosphonate were administrated in many subjects.

Vertebral morphology after fracture was assessed. The results showed that non-weight-bearing $\mathrm{VHa}$ in both groups had significantly decreased at 12 weeks compared with baseline, and VHa at 12 weeks had decreased to nearly the weight-bearing VHa observed at baseline (Table 2). At 12 weeks, weight-bearing $\mathrm{VHa}$ in both groups had also significantly decreased, compared with baseline. $\Delta \mathrm{VHa}$, indicating vertebral stability, tended to be lower in the TPTD group at 12 weeks ( $p=0.17$ ), but there were no significant difference between groups. Although not significant, the rate of bony union tended to be higher in the TPTD group (control, $48 \%$; TPTD, $68 \%$; $p=0.16$ ).
In accordance with the diagnostic criteria for primary osteoporosis [18], subjects with severe osteoporosis were categorized as having a 'lumbar spine BMD T-score below -3.0 ,' 'at least two prevalent OVFs', or 'a prevalent OVF with SQ grade 3 vertebral deformity.' Further analysis was performed on subjects with severe osteoporosis as described above. Fifteen subjects in the control group and 16 subjects in the TPTD group were found to have severe osteoporosis (Table 3). Baseline characteristics in both groups with severe osteoporosis indicated that the TPTD group had significantly lower lumbar spine BMD Tscores, despite significantly lower mean age (Table 3). At baseline, there were no significant differences in the rates of osteoporosis treatment between groups.

$\mathrm{VHa}$ (weight-bearing position) for subjects with severe osteoporosis in both groups had significantly decreased at 12 weeks, compared with baseline (Table 4). Progressive vertebral collapse was significantly more common in the control group, compared with the TPTD group (control, 26\%; TPTD, 12\%; $p=0.03$ ). Moreover, despite no significant differences in $\triangle \mathrm{VHa}$ between groups at baseline, $\Delta \mathrm{VHa}$ was significantly smaller in the TPTD group than in the control group at 12 weeks (control: mean \pm SE, $3.1 \pm 0.6 \mathrm{~mm}$; TPTD: mean \pm SE, $1.4 \pm 0.5 \mathrm{~mm}$;

Table 1. Characteristics of study subjects

\begin{tabular}{|c|c|c|c|}
\hline Characteristic & $\begin{array}{l}\text { Control group } \\
\qquad(n=23)\end{array}$ & $\begin{array}{c}\text { TPTD } \\
\text { group }(n=25)\end{array}$ & $p$-value \\
\hline Age (yr) & $79.5 \pm 1.6$ & $77.8 \pm 1.1$ & 0.23 \\
\hline Body mass index $\left(\mathrm{kg} / \mathrm{m}^{2}\right)$ & $21.5 \pm 0.8$ & $21.2 \pm 0.7$ & 0.85 \\
\hline Gender (male:female) & $6: 17$ & $2: 23$ & 0.09 \\
\hline Rates of osteoporosis treatment at baseline (\%) & 17 & 40 & 0.09 \\
\hline Presence of prevalent OVF (\%) & 48 & 56 & 0.57 \\
\hline Level of the injured vertebra (T10-L2, \%) & 83 & 80 & 1.00 \\
\hline No. of prevalent OVF confirmed by magnetic resonance imaging images (T4-L4) & $0.9 \pm 0.3$ & $1.1 \pm 0.3$ & 0.65 \\
\hline No. of prevalent OVFs with severe vertebral deformity (semi-quantitative method grade 3) & $0.2 \pm 0.1$ & $0.6 \pm 0.2$ & 0.11 \\
\hline Lumbar spine BMD T-score & $-2.6 \pm 0.3$ & $-3.1 \pm 0.3$ & 0.22 \\
\hline Femoral neck BMD T-score & $-2.3 \pm 0.2$ & $-2.4 \pm 0.2$ & 0.67 \\
\hline Estimated glomerular filtration rate $\left(\mathrm{mL} / \mathrm{min} / 1.73 \mathrm{~m}^{2}\right)$ & $61.0 \pm 3.6$ & $62.5 \pm 3.0$ & 0.42 \\
\hline Intact N-terminal propeptide of type I procollagen $(\mu \mathrm{g} / \mathrm{L})$ & $54.3 \pm 7.8$ & $46.4 \pm 4.0$ & 0.35 \\
\hline Tartrate-resistant acid phosphatase 5b (mU/dL) & $455 \pm 37$ & $389 \pm 34$ & 0.18 \\
\hline Parathyroid hormone $(\mathrm{pg} / \mathrm{mL})$ & $46.5 \pm 4.7$ & $35.1 \pm 3.7$ & 0.16 \\
\hline
\end{tabular}

Values are presented as mean \pm standard error or number. Statistical analysis was conducted using Mann-Whitney U-test or chi-square test or Fisher's exact test: $p<0.05$ was considered a statistically significant difference.

TPTD, teriparatide; OVF, osteoporotic vertebral fracture; BMD, bone mineral density. 
Table 2. Changes of VHa and collapse rate in subjects with acute osteoporotic vertebral fracture

\begin{tabular}{|c|c|c|c|}
\hline Variable & Control group (n=23) & TPTD group (n=25) & $p$-value \\
\hline \multicolumn{4}{|l|}{ Baseline } \\
\hline Non-weight-bearing VHa (mm) & $24.5 \pm 2.0$ & $24.3 \pm 0.9$ & 0.90 \\
\hline Weight-bearing VHa (mm) & $17.7 \pm 1.4$ & $18.3 \pm 1.0$ & 0.76 \\
\hline$\Delta \mathrm{VHa}(\mathrm{mm})$ & $6.8 \pm 0.6$ & $5.8 \pm 0.6$ & 0.33 \\
\hline \multicolumn{4}{|l|}{$12 w k$} \\
\hline Non-weight-bearing VHa (mm) & $17.4 \pm 1.5^{\text {a) }}$ & $18.0 \pm 1.0^{a)}$ & 0.85 \\
\hline Weight-bearing VHa (mm) & $15.4 \pm 1.6^{b l}$ & $16.0 \pm 1.1^{b)}$ & 0.81 \\
\hline$\Delta \mathrm{VHa}(\mathrm{mm})$ & $2.7 \pm 0.5$ & $2.2 \pm 0.5$ & 0.17 \\
\hline Vertebral collapse rate (\%) & $19.9 \pm 4.4$ & $14.5 \pm 4.0$ & 0.38 \\
\hline Bony union rate $(\Delta \mathrm{VHa}<2 \mathrm{~mm})$ & 47.8 & 68.0 & 0.16 \\
\hline
\end{tabular}

Values are presented as mean \pm standard error or $\% . \Delta \mathrm{VHa}=\mathrm{VHa}$ (supine position)-VHa (weight-bearing position); vertebral collapse rate=VHa (weight-bearing position, baseline)-VHa (weight-bearing position, 12 weeks after starting treatment)/VHa (weight-bearing position, baseline). Statistical analysis between the control group and the TPTD group was conducted using Mann-Whitney U-test or chi-square test: $p<0.05$ was considered a statistically significant difference.

VHa, vertebral height at the anterior location; TPTD, teriparatide.

${ }^{\text {a) }}$ Significant difference of $\mathrm{VHa}$ in non-weight-bearing position (baseline vs. 12 weeks). ${ }^{\text {b) }}$ Significant difference of VHa in weight-bearing position (baseline vs. 12 weeks).

Table 3. Characteristics of study subjects with severe osteoporosis

\begin{tabular}{|c|c|c|c|}
\hline Characteristic & Control group ( $n=15)$ & TPTD group $(n=16)$ & $p$-value \\
\hline Age (yr) & $81.6 \pm 1.5$ & $76.8 \pm 1.4$ & $0.03^{*}$ \\
\hline Body mass index $\left(\mathrm{kg} / \mathrm{m}^{2}\right)$ & $20.6 \pm 1.0$ & $20.9 \pm 0.8$ & 0.58 \\
\hline Gender (male:female) & $2: 13$ & $1: 15$ & 0.60 \\
\hline Rates of osteoporosis treatment at baseline (\%) & 20.0 & 31.3 & 0.69 \\
\hline Presence of prevalent OVF $(\%)$ & 66.7 & 68.8 & 1.00 \\
\hline Level of the injured vertebra (T10-L2, \%) & 93 & 88 & 0.71 \\
\hline No. of prevalent OVF confirmed by magnetic resonance imaging images (T4-L4) & $1.2 \pm 0.4$ & $1.2 \pm 0.3$ & 0.92 \\
\hline Lumbar spine bone mineral density T-score & $-3.3 \pm 0.3$ & $-4.0 \pm 0.3$ & $0.04^{*}$ \\
\hline Estimated glomerular filtration rate $\left(\mathrm{mL} / \mathrm{min} / 1.73 \mathrm{~m}^{2}\right)$ & $58.9 \pm 3.0$ & $63.2 \pm 4.4$ & 0.32 \\
\hline Intact N-terminal propeptide of type I procollagen ( $\mu \mathrm{g} / \mathrm{L}$ ) & $52.2 \pm 9.0$ & $53.9 \pm 5.3$ & 0.34 \\
\hline Tartrate-resistant acid phosphatase $5 b(\mathrm{mU} / \mathrm{dL})$ & $486 \pm 47$ & $432 \pm 46$ & 0.33 \\
\hline Parathyroid hormone $(\mathrm{pg} / \mathrm{mL})$ & $44.8 \pm 5.6$ & $32.4 \pm 2.1$ & 0.07 \\
\hline
\end{tabular}

Values are presented as mean \pm standard error or \%. Statistical analysis was conducted using Mann-Whitney U-test, chi-square test, or Fisher's exact test: $p<0.05$ was considered a statistically significant difference.

TPTD, teriparatide; OVF, osteoporotic vertebral fracture.

* $p<0.05$ (significant difference between control and TPTD group).

$p=0.02$ ) (Table 4). The rate of bony union was significantly higher in the TPTD group (control, $40 \%$; TPTD, $81 \%$; $p=0.03$ ).

\section{Discussion}

We investigated the effects of once-weekly TPTD admin- istration on vertebral stability and bony union after acute OVF. Previous studies of bony union after acute OVF had the following issues: (1) methods to assess bony union were often qualitative, and definitions of bony union differed from study to study; (2) previous studies failed to exclude cases of stable OVF, in which bony union is relatively common, regardless of the therapy used. To the best 
Table 4. Changes of VHa and collapse rate in subjects with acute osteoporotic vertebral fracture accompanying with severe osteoporosis

\begin{tabular}{|c|c|c|c|}
\hline Variable & Control group $(\mathrm{n}=15)$ & TPTD group $(\mathrm{n}=16)$ & $p$-value \\
\hline \multicolumn{4}{|l|}{ Baseline } \\
\hline Non-weight-bearing VHa (mm) & $24.4 \pm 1.4$ & $24.4 \pm 1.2$ & 0.74 \\
\hline Weight-bearing VHa (mm) & $18.0 \pm 1.6$ & $18.7 \pm 1.2$ & 0.43 \\
\hline$\Delta \mathrm{VHa}(\mathrm{mm})$ & $6.7 \pm 0.7$ & $5.6 \pm 0.6$ & 0.24 \\
\hline \multicolumn{4}{|l|}{12 wk } \\
\hline Non-weight-bearing VHa (mm) & $18.0 \pm 1.7^{\text {a) }}$ & $18.2 \pm 1.4^{\text {a) }}$ & 0.85 \\
\hline Weight-bearing VHa (mm) & $15.0 \pm 1.6^{b)}$ & $16.7 \pm 1.5^{\mathrm{b})}$ & 0.81 \\
\hline$\Delta \mathrm{VHa}(\mathrm{mm})$ & $3.1 \pm 0.6$ & $1.4 \pm 0.5$ & $0.02^{*}$ \\
\hline Vertebral collapse rate (\%) & $25.8 \pm 4.3$ & $12.0 \pm 4.1$ & $0.03^{*}$ \\
\hline Bony union rate $(\Delta \mathrm{VHa}<2 \mathrm{~mm})$ & 40.0 & 81.3 & $0.03^{*}$ \\
\hline
\end{tabular}

Values are presented as mean \pm standard error or $\% . \Delta \mathrm{VHa}=\mathrm{VHa}$ (supine position)-VHa (weight-bearing position); vertebral collapse rate=VHa (weight-bearing position, baseline)-VHa (weight-bearing position, 12 weeks after starting treatment)/VHa (weight-bearing position, baseline). Statistical analysis between the control group and the TPTD group was conducted using Mann-Whitney U-test or chi-square test: $p<0.05$ was considered a statistically significant difference.

$\mathrm{VHa}$, vertebral height at the anterior location; TPTD teriparatide.

${ }^{*} p<0.05$ (significant difference between control and TPTD group). ${ }^{a)}$ Significant difference of VHa in non-weight-bearing position (baseline vs. 12 weeks). ${ }^{\text {b) }}$ Significant difference of $\mathrm{VHa}$ in weight-bearing position (baseline vs. 12 weeks).

of our knowledge, this is the first study to investigate the effect of TPTD on acute OVF using quantitative criteria for the evaluation of bony union after the exclusion of subjects with stable acute OVF. The results of the present study indicated that the effects of once-weekly TPTD administration promoted bony union in fractured vertebrae in patients with severe osteoporosis. This agent therefore appears to prevent further vertebral collapse and contributes to early vertebral stability.

Previous studies regarding bony union after acute OVF had a serious issue: methods used to assess bony union were often qualitative and definitions of bony union differed from study to study. Several authors reported that $\Delta \mathrm{VHa}$ of $2 \mathrm{~mm}$ is the most reasonable cutoff value for diagnosis of acute OVF using lateral radiographs obtained in weight- and non-weight-bearing positions $[12,13]$. Sato et al. [16] defined union as the absence of abnormal motion $(\triangle \mathrm{VHa}>1 \mathrm{~mm})$ and reported that the rate of bony union 3 months after conservative therapy for acute OVF was $42 \%$ (in the case of $\triangle \mathrm{VHa}>2 \mathrm{~mm}$, the rate of bony union was $80 \%)$. Niimi et al. [13] showed that precision errors associated with measurements of $\mathrm{VHa}$ corresponded to standard deviation of $1.2 \mathrm{~mm}$, and SE VHa and $\triangle \mathrm{VHa}$ (intraobserver) were $0.48 \mathrm{~mm}$ and $0.66 \mathrm{~mm}$, respectively. In order to ensure the accuracy of measurements obtained with plain radiography, we defined bony union as the absence of a vertebral cleft or abnormal mo- tion $(\triangle \mathrm{VHa}>2 \mathrm{~mm}) 3$ months after starting treatment.

Few previous reports have investigated the effects of TPTD for fracture repair in humans [9-11], and the effects of TPTD for fracture repair remain controversial $[7,8]$. To the best of our knowledge, no previous study published in English has investigated the effect of once-weekly TPTD administration on bony union after acute OVF. One such study has been published in the Japanese language [19]. The study reported that, 12 weeks after treatment with anti-osteoporosis drugs, bony union was significantly higher in the once-weekly TPTD group than in the bisphosphonate group (at 73\% versus 45\%) [19]. Our study results in subjects with severe osteoporosis (control, 40\%; TPTD, $81 \%$ ) are compatible with the rate of bony union reported in their study.

When we restricted our investigation to subjects with severe osteoporosis, the $\triangle \mathrm{VHa}$ was significantly smaller in the TPTD group than in the control group at 12 weeks (control: mean \pm SE, $3.1 \pm 0.6 \mathrm{~mm}$; TPTD: mean $\pm \mathrm{SE}$, $1.4 \pm 0.5$ $\mathrm{mm} ; p=0.02$ ). In addition, progressive vertebral collapse was significantly less frequent in the TPTD group, compared with the control group (control, 26\%; TPTD, 12\%; $p=0.03$ ). In control subjects with severe osteoporosis, we observed a vicious cycle of further deformity of injured vertebrae during the post-fracture course. This cycle resulted in increased $\triangle \mathrm{VHa}$, persistent instability, and delayed union. However, when compared with the control 
group, the TPTD group showed greater suppression in progressive vertebral deformity during the post-fracture course, leading to more rapid achievement of vertebral stability (smaller $\Delta \mathrm{VHa}$ ) and a higher rate of bony union. This result is impressive, given the fact that the TPTD group had significantly lower lumbar-spine BMD, conditions which increase the likelihood of progressive vertebral deformity of the fractured vertebra. Taken together, these results indicate that TPTD increases the rate of vertebral bony union by suppressing vertebral collapse and promoting vertebral stability. Previous studies have reported that TPTD prevents the collapse of fractured vertebral bodies after acute OVF [20-22]. Tsuchie et al. [20] conducted a study similar to ours, in which TPTD was administered to subjects with acute OVF for 12 weeks. Tsuchie et al. [20] reported that, at 4 weeks after TPTD administration, TPTD was effective in preventing collapse of the injured vertebra. The authors suggested the possibility that TPTD administration promoted cartilage formation through endochondral ossification, which prevented early vertebral collapse [22].

The present study has several limitations. As the first limitation, this study was not a double-blind, randomized prospective study. To investigate the issues more precisely, a double-blind, randomized prospective study should be performed. However, in the design of such a study, the control group would be required to interrupt antiosteoporotic drugs for a fixed period of time. Termination of antiosteoporotic drugs could cause additional vertebral fractures and would therefore be ethically unacceptable. The second limitation was the fact that the number of subjects in both groups was relatively small. Unlike previous studies, we excluded cases of stable acute OVF that showed no or little change in $\triangle \mathrm{VHa}$, thereby limiting the patient population to those with vertebral instability and decreasing the number of subjects included in the study. The third limitation was the fact that a study period of 3 months is relatively brief. However, pain and other symptoms caused by acute OVF normally resolve within 3 months. Vertebral collapse is most common within 3 months of injury [23]; it is therefore reasonable to investigate the effects of TPTD on vertebral fractures within a 3-month period.

\section{Conclusions}

The results of the present study indicate that once-weekly administration of TPTD promotes bony union of fractured vertebra in patients with severe osteoporosis. This approach to treatment appears to promote the stability of fractured vertebrae by preventing further vertebral collapse during the immediate post-injury period.

\section{Conflict of Interest}

No potential conflict of interest relevant to this article was reported.

\section{References}

1. Yamaguchi T, Sugimoto T, Yamauchi M, Matsumori Y, Tsutsumi M, Chihara K. Multiple vertebral fractures are associated with refractory reflux esophagitis in postmenopausal women. J Bone Miner Metab 2005;23:36-40.

2. Harrison RA, Siminoski K, Vethanayagam D, Majumdar SR. Osteoporosis-related kyphosis and impairments in pulmonary function: a systematic review. J Bone Miner Res 2007;22:447-57.

3. Silverman SL, Minshall ME, Shen W, Harper KD, Xie S; Health-Related Quality of Life Subgroup of the Multiple Outcomes of Raloxifene Evaluation Study. The relationship of health-related quality of life to prevalent and incident vertebral fractures in postmenopausal women with osteoporosis: results from the Multiple Outcomes of Raloxifene Evaluation Study. Arthritis Rheum 2001;44:2611-9.

4. Matsumoto T, Hoshino M, Tsujio T, et al. Prognostic factors for reduction of activities of daily living following osteoporotic vertebral fractures. Spine (Phila Pa 1976) 2012;37:1115-21.

5. Takahashi S, Hoshino M, Tsujio T, et al. Risk factors for cognitive decline following osteoporotic vertebral fractures: a multicenter cohort study. J Orthop Sci 2017;22:834-9.

6. Kashii M, Yamazaki R, Yamashita T, et al. Surgical treatment for osteoporotic vertebral collapse with neurological deficits: retrospective comparative study of three procedures: anterior surgery versus posterior spinal shorting osteotomy versus posterior spinal fusion using vertebroplasty. Eur Spine J 2013;22:163342.

7. Lou S, Lv H, Wang G, et al. The effect of teriparatide on fracture healing of osteoporotic patients: a meta- 
analysis of randomized controlled trials. Biomed Res Int 2016;2016:6040379.

8. Shi Z, Zhou H, Pan B, et al. Effectiveness of teriparatide on fracture healing: a systematic review and meta-analysis. PLoS One 2016;11:e0168691.

9. Aspenberg P, Genant HK, Johansson T, et al. Teriparatide for acceleration of fracture repair in humans: a prospective, randomized, double-blind study of 102 postmenopausal women with distal radial fractures. J Bone Miner Res 2010;25:404-14.

10. Kanakaris NK, West RM, Giannoudis PV. Enhancement of hip fracture healing in the elderly: evidence deriving from a pilot randomized trial. Injury 2015;46:1425-8.

11. Johansson T. PTH 1-34 (teriparatide) may not improve healing in proximal humerus fractures: a randomized, controlled study of 40 patients. Acta Orthop 2016;87:79-82.

12. Kawasaki M, Tsuboya H, Kiyasu K, Ueta E, Takemasa $\mathrm{R}$, Tani T. Diagnostic accuracy of the plain radiography on sitting-supine position for fresh vertebral fracture (in Japanese). Kossetsu (Fract) 2008;30:26973.

13. Niimi R, Kono T, Nishihara A, et al. Efficacy of the dynamic radiographs for diagnosing acute osteoporotic vertebral fractures. Osteoporos Int 2014;25:60512.

14. Kawaguchi S, Horigome K, Yajima H, Oda T, Kii Y. Comparative supine-sitting lateral radiographs identifying incident osteoporotic vertebral fractures. Eur Orthop Traumatol 2011;1:157-62.

15. McKiernan F, Jensen R, Faciszewski T. The dynamic mobility of vertebral compression fractures. J Bone Miner Res 2003;18:24-9.

16. Sato K, Yamahiro M, Kasama F, Matuda M. Conservative treatment of osteoporotic vertebral frac- tures: patient management in a recovery rehabilitation ward (in Japanese). Seikeigeka (Orthop Surg) 2013;64:1247-54.

17. Genant HK, Wu CY, van Kuijk C, Nevitt MC. Vertebral fracture assessment using a semiquantitative technique. J Bone Miner Res 1993;8:1137-48.

18. Soen S, Fukunaga M, Sugimoto T, et al. Diagnostic criteria for primary osteoporosis: year 2012 revision. J Bone Miner Metab 2013;31:247-57.

19. Shigenobu K, Kanayama M, Ohwa F, et al. Effects of anti-osteoporosis drugs for pain, activities of daily living, quality of life, bone metabolism and bony union in patients with acute osteoporotic vertebral fractures: comparison between once weekly teriparatide and bisphosphonate (in Japanese). Osteoporos Jpn 2014;22:117-21.

20. Tsuchie H, Miyakoshi N, Kasukawa Y, et al. The effect of teriparatide to alleviate pain and to prevent vertebral collapse after fresh osteoporotic vertebral fracture. J Bone Miner Metab 2016;34:86-91.

21. Fahrleitner-Pammer A, Langdahl BL, Marin F, et al. Fracture rate and back pain during and after discontinuation of teriparatide: 36-month data from the European Forsteo Observational Study (EFOS). Osteoporos Int 2011;22:2709-19.

22. Hadji P, Zanchetta JR, Russo L, et al. The effect of teriparatide compared with risedronate on reduction of back pain in postmenopausal women with osteoporotic vertebral fractures. Osteoporos Int 2012;23:2141-50.

23. Goldstein S, Smorgick Y, Mirovsky Y, Anekstein Y, Blecher R, Tal S. Clinical and radiological factors affecting progressive collapse of acute osteoporotic compression spinal fractures. J Clin Neurosci 2016;31:122-6. 